\title{
A recursive dynamics algorithm for soft robotic manipulators made of viscoelastic material
}

\author{
$\underline{\text { Makoto Iwamura }}^{1}$, Kento Hirata $^{1}$, Yoshiki Maeda $^{1}$, Kyuji Oto $^{1}$ \\ ${ }^{1}$ Department of Mechanical Engineering \\ Fukuoka University \\ 8-19-1 Nanakuma, Jonan, 814-0180 Fukuoka, Japan \\ iwamura@fukuoka-u.ac.jp
}

\begin{abstract}
In recent years, research and development of robots that exist in the same space as humans and can collaborate with humans have been actively carried out. If the body of a robot is made of a hard material, it may cause injury. Therefore, attempts have been made to make a robot with a soft body using rubber or resin. In order to accelerate such research on soft robotics, it is necessary to establish fast and stable simulation algorithm for robots containing viscoelastic bodies such as rubber and resin. Therefore, in this study, we consider to approximate viscoelastic bodies with finite rigid body segments and connect them with joints and linear viscoelastic elements such as Voigt model and Maxwell model to approximate viscoelastic properties. The recursive dynamics algorithm is used to speed up the calculation, and the generalization- $\alpha$ method is used to stabilize the numerical integration. In particular, we propose a new method on how to incorporate the Maxwell model into recursive dynamics algorithm and generalization- $\alpha$ method. The effectiveness of the proposed method is confirmed by some numerical examples.
\end{abstract}

Keywords: Soft robotics, Recursive algorithm, Viscoelastic material, Maxwell model.

\section{INTRODUCTION}

In the past, industrial robots had to be fenced for safety and completely separate the working range of humans and robots. However, due to the relaxation of the law, robots that meet certain conditions can now exist and collaborate in the same space as humans without being surrounded by fences. Furthermore, in recent years, many robots for long-term care and home use have been developed, and robots are deeply entering the living space of human beings. If the body of a robot that collaborates with a person is made of a hard material, it may cause serious injury when it comes into contact with a person. Therefore, attempts have been made to give the robot a soft body using rubber or resin. In order to accelerate the research and development of such soft robotics [1], it is necessary to establish a high-speed and stable dynamical simulation technology for robot systems including viscoelastic bodies such as rubber and resin. So far, research has been conducted to describe viscoelastic bodies by the nonlinear finite element method and incorporate them into multibody dynamics analysis [2,3], however there is a problem that the theory is difficult and the calculation time is enormous.

Therefore, in this study, we consider to approximate viscoelastic bodies with finite rigid body segments and connect them with joints and linear viscoelastic elements such as Voigt model and Maxwell model to express viscoelastic properties. The recursive dynamics algorithm [4] is used to speed up the calculation, and the generalized- $\alpha$ method [5] is used to stabilize the numerical integration. In this paper, we examine how to incorporate the Maxwell model into the recursive dynamics algorithm and the generalized $\alpha$ method, and propose a new method. We perform a dynamical simulation of a 2-link manipulator composed of rubber links to confirm the effectiveness of the proposed method. 


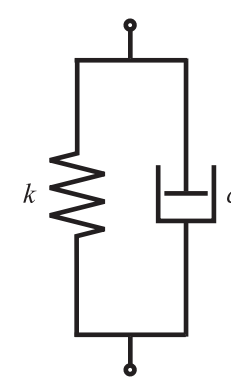

(a) Voigt model

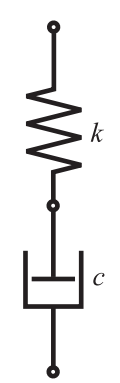

(b) Maxwell model

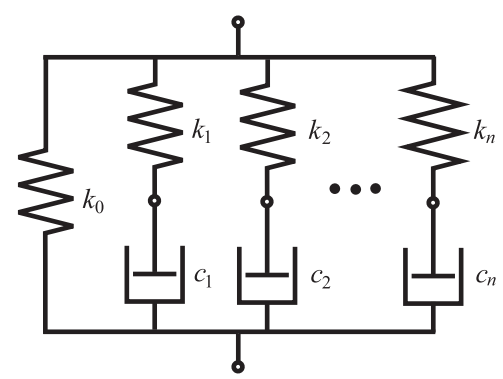

(c) Generalized Maxwell model

Figure 1. Linear viscoelastic model

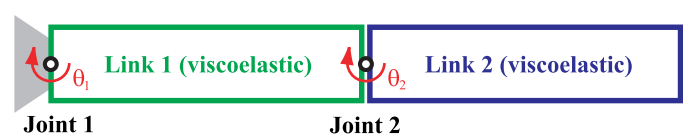

Figure 2. 2-link manipulator made of silicone rubber

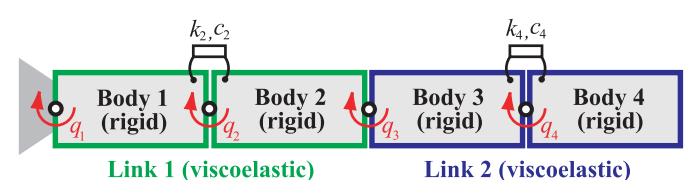

Figure 3. Finite segment approximation

\section{MODEL OF SOFT ROBOT SYSTEM INCLUDING VISCOELASTIC BODY}

The nonlinear finite element method is sometimes used to model a viscoelastic body, but it is not suitable for real-time simulation due to the large amount of calculation. On the other hand, a method of approximately expressing viscoelasticity by using the Voigt model, Maxwell model, generalized Maxwell model, etc., which combine the spring element and damper element as shown in Fig. 1, is also used. In this study, individual viscoelastic bodies included in a robot system are approximated by multiple rigid body elements connected by rotational joints or prismatic joints, and viscoelastic properties are expressed by adding spring elements and damper elements to the joints.

As an example, consider a 2-link manipulator composed of rubber links as shown in Fig. 2. Here, one link is approximated by two rigid body segments, and it is assumed that the axial deformation is negligible, and the bending deformation is expressed by connecting with a rotating joint. Then, by adding an appropriate rotary spring and rotary damper to the joint, the viscoelastic property of the link is approximately expressed. As a result, the system in Fig. 2 can be modeled as in Fig. 3. In the following, the relative angular displacement of the joint $i$ is $q_{i}$, the spring constant of the spring added to the joint $i$ is $k_{i}$, and the viscous damping coefficient of the damper is $c_{i}$. The length of the body $i$ is $l_{i}$, the mass is $m_{i}$, and the moment of inertia around the center of gravity is $I_{i}$.

In this study, as a stepping stone for the construction of a general calculation method, we formulate an algorithm that can perform high-speed and stable calculations for the system that was modeled as an open-loop rigid multibody system as shown in Fig. 4 and the Voigt model or Maxwell model as shown in Fig. 5 was added to the joints. When a soft robot system containing a viscoelastic body is modeled as a rigid multibody system as shown in Fig. 4, a high-speed recursive algorithm can be applied to dynamic calculations. In addition, the generalized- $\alpha$ method, which has excellent stability and allows a large time step size, is used for numerical integration.

\section{RECURSIVE DYNAMICS ALGORITHM}

In this section, the recursive dynamics algorithm [4] is extended to a rigid multibody system in which rigid bodies are connected in series by rotating joints as shown in Fig. 4 and a Voigt model or Maxwell model is added to the joints as shown in Fig. 5. It is assumed that the total number of bodies is $N$, including the rigid body elements introduced to approximate the viscoelastic body. Here, the body $i$ coordinate system $\Sigma_{i}$ is defined so as to match the joint $i$ on the root side. Let 


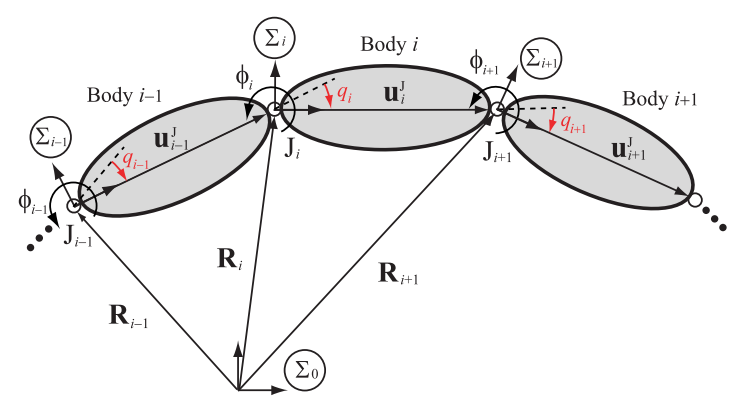

Figure 4. Relationship between neighboring bodies

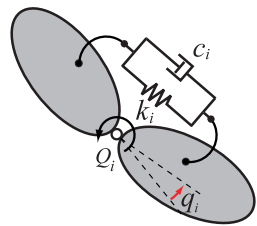

(a) Voigt model

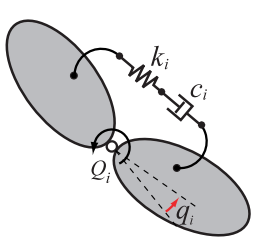

(b) Maxwell model
Figure 5. Viscoelastic model added to joints

the vector from the origin of the absolute coordinate system $\Sigma_{0}$ to the origin of $\Sigma_{i}$ be $\boldsymbol{R}_{i}$, and the angle of $\Sigma_{i}$ with respect to $\Sigma_{0}$ be $\phi_{i}$. Then, the velocity relationship and acceleration relationship between the adjacent body $i$ and body $i-1$ can be expressed by the following equations.

$$
\begin{aligned}
& \boldsymbol{v}_{i}=\boldsymbol{D}_{i} \boldsymbol{v}_{i-1}+\boldsymbol{J}_{i} \dot{q}_{i} \\
& \boldsymbol{a}_{i}=\boldsymbol{D}_{i} \boldsymbol{a}_{i-1}+\boldsymbol{J}_{i} \ddot{q}_{i}+\boldsymbol{\beta}_{i}
\end{aligned}
$$

where $\boldsymbol{v}_{i}=\left[\begin{array}{ll}\dot{\boldsymbol{R}}_{i}^{T} & \dot{\phi}_{i}\end{array}\right]^{T}, \boldsymbol{a}_{i}=\left[\ddot{\boldsymbol{R}}_{i}^{T} \ddot{\phi}_{i}\right]^{T}, \boldsymbol{J}_{i}$ is the Jacobian matrix which represents the effect of joint velocity $\dot{q}_{i}$ on generalized velocity $\boldsymbol{v}_{i}, \boldsymbol{D}_{i}=\operatorname{diag} .\left[\boldsymbol{A}_{i}, 1\right], \boldsymbol{A}_{i}$ is the rotation matrix from $\Sigma_{i}$ to $\Sigma_{i-1}$, and $\boldsymbol{\beta}_{i}=\dot{\boldsymbol{D}}_{i} \boldsymbol{v}_{i-1}+\dot{\boldsymbol{J}}_{i} \dot{q}_{i}$.

On the other hand, the equations of motion of the body $i$ can be expressed as follows.

$$
\boldsymbol{M}_{i} \boldsymbol{a}_{i}+\boldsymbol{h}_{i}=\boldsymbol{Q}_{i}
$$

where $\boldsymbol{M}_{i}$ is the generalized mass matrix, $\boldsymbol{h}_{i}$ is the quadratic velocity vector, $\boldsymbol{Q}_{i}$ is the generalized force vector. The generalized force $\boldsymbol{Q}_{i}$ acting on the body $i$ is divided into three forces, that is, the generalized force $\boldsymbol{Q}_{i}^{J}$ received from the body $i-1$ via the joint $i$, the reaction force of the generalized force $Q_{i+1}^{J}$ exerted by the body $i$ on the body $i+1$ via the joint $i+1$, and the other generalized forces $\boldsymbol{Q}_{i}^{O}$. Therefore, it can be expressed as $\boldsymbol{Q}_{i}=\boldsymbol{Q}_{i}^{J}+\boldsymbol{Q}_{i}^{O}-\boldsymbol{D}_{i+1}^{T} \boldsymbol{Q}_{i+1}^{J}$. By substituting this into Eq. (3), solving for $\boldsymbol{Q}_{i}^{J}$, and taking the inner product with $\boldsymbol{J}_{i}$, then the joint driving torque $\tau_{i}$ can be obtained.

$$
\begin{aligned}
\boldsymbol{Q}_{i}^{J} & =\boldsymbol{M}_{i} \boldsymbol{a}_{i}+\boldsymbol{h}_{i}-\boldsymbol{Q}_{i}^{O}+\boldsymbol{D}_{i+1}^{T} \boldsymbol{Q}_{i+1}^{J} \\
\tau_{i} & =\boldsymbol{J}_{i}^{T} \boldsymbol{Q}_{i}^{J}+\bar{Q}_{i}
\end{aligned}
$$

where $\bar{Q}_{i}$ is the reaction torque of $Q_{i}$ applied by the Voigt model or Maxwell model added to the joint $i$.

Inverse dynamics calculation can be executed by calculating the equations (1) and (2) from the root $i=1$ to the tip $i=N$, and then calculating the equations (4) and (5) from the tip $i=N$ to the root $i=1$. By repeatedly using this inverse dynamics calculation, the mass matrix $\boldsymbol{M}$, and the centrifugal, Coriolis and gravitational force $\boldsymbol{h}$ of the following equations of motion in minimal form can be calculated efficiently.

$$
\boldsymbol{M}(\boldsymbol{q}) \ddot{\boldsymbol{q}}+\boldsymbol{h}(\boldsymbol{q}, \dot{\boldsymbol{q}})=\boldsymbol{\tau}
$$

\section{NUMERICAL INTEGRATION METHOD}

Numerical integration methods are roughly divided into explicit methods and implicit methods. Since a system containing a viscoelastic body is generally a stiff system, it is necessary to make the time step $h$ extremely small when using the explicit method in which the stable range is limited, which requires a huge amount of calculation time. On the other hand, the implicit method is 
excellent in stability, and since the time step $h$ can be taken large, the solution can be obtained in a short calculation time. Here, we use the generalized- $\alpha$ method [5], which is a single-step implicit method. The implicit method requires iterative calculations at each step, and at that time the Jacobian matrix must be calculated. Hence, we propose a method for calculating the Jacobian matrix strictly and at high speed using the recursive dynamics algorithm formulated in section 3 to shorten the calculation time.

\subsection{Generalized- $\alpha$ method}

Let us divide the interval $t \in\left[0, t_{f}\right]$ for the solution into $0=t_{0}<t_{1}<\cdots<t_{p-1}<t_{p}=t_{f}$ and express the value of $\boldsymbol{q}(t)$ at time $t_{n}$ as $\boldsymbol{q}_{n}$. We also introduce an auxiliary variable vector $\boldsymbol{a}_{n}$ of the same dimension as the acceleration that defined by the following recurrence formula.

$$
\left(1-\alpha_{m}\right) \boldsymbol{a}_{n+1}+\alpha_{m} \boldsymbol{a}_{n}=\left(1-\alpha_{f}\right) \ddot{\boldsymbol{q}}_{n+1}+\alpha_{f} \ddot{\boldsymbol{q}}_{n}, \boldsymbol{a}_{0}=\ddot{\boldsymbol{q}}_{0}
$$

Then, we use the following formula in which $\ddot{\boldsymbol{q}}_{n}$ and $\ddot{\boldsymbol{q}}_{n+1}$ in Newmark's integral formula are replaced with $\boldsymbol{a}_{n}$ and $\boldsymbol{a}_{n+1}$, respectively.

$$
\begin{aligned}
& \dot{\boldsymbol{q}}_{n+1}=\dot{\boldsymbol{q}}_{n}+h(1-\gamma) \boldsymbol{a}_{n}+h \gamma \boldsymbol{a}_{n+1} \\
& \boldsymbol{q}_{n+1}=\boldsymbol{q}_{n}+h \dot{\boldsymbol{q}}_{n}+h^{2}\left(\frac{1}{2}-\beta\right) \boldsymbol{a}_{n}+h^{2} \beta \boldsymbol{a}_{n+1}
\end{aligned}
$$

From equations (7) to (9), the following relationship can be obtained.

$$
\begin{aligned}
& \dot{\boldsymbol{q}}_{n+1}=\frac{\gamma}{h \beta}\left(\boldsymbol{q}_{n+1}-\boldsymbol{q}_{n}\right)+\left(1-\frac{\gamma}{\beta}\right) \dot{\boldsymbol{q}}_{n}+h\left(1-\frac{\gamma}{2 \beta}\right) \boldsymbol{a}_{n} \\
& \ddot{\boldsymbol{q}}_{n+1}=\frac{1-\alpha_{m}}{h^{2} \beta\left(1-\alpha_{f}\right)}\left\{\boldsymbol{q}_{n+1}-\boldsymbol{q}_{n}-h \dot{\boldsymbol{q}}_{n}-h^{2}\left(\frac{1}{2}-\beta\right) \boldsymbol{a}_{n}\right\}+\frac{\alpha_{m} \boldsymbol{a}_{n}-\alpha_{f} \ddot{\boldsymbol{q}}_{n}}{1-\alpha_{f}}
\end{aligned}
$$

From equations (10) and (11), it can be seen that $\dot{\boldsymbol{q}}_{n+1}$ and $\ddot{\boldsymbol{q}}_{n+1}$ are only functions of $\boldsymbol{q}_{n+1}$, if $\boldsymbol{q}_{n}$, $\dot{\boldsymbol{q}}_{n}, \ddot{\boldsymbol{q}}_{n}$ and $\boldsymbol{a}_{n}$ at time $t$ are known. If the above equations are partially differentiated with respect to $\boldsymbol{q}_{n+1}$, the following relationships can be obtained.

$$
\frac{\partial \dot{\boldsymbol{q}}_{n+1}}{\partial \boldsymbol{q}_{n+1}}=\gamma^{\prime} \boldsymbol{E}, \frac{\partial \ddot{\boldsymbol{q}}_{n+1}}{\partial \boldsymbol{q}_{n+1}}=\beta^{\prime} \boldsymbol{E}
$$

where $\gamma^{\prime} \equiv \gamma /(h \beta), \beta^{\prime} \equiv\left(1-\alpha_{m}\right) /\left\{h^{2} \beta\left(1-\alpha_{f}\right)\right\}$.

The residual of the equations of motion in minimal form (6) at time $t=t_{n+1}$ is written by explicitly expressing $\dot{\boldsymbol{q}}_{n+1}$ and $\ddot{\boldsymbol{q}}_{n+1}$ are functions of $\boldsymbol{q}_{n+1}$ as follows.

$$
\boldsymbol{e}\left(\boldsymbol{q}_{n+1}\right) \equiv \boldsymbol{M}\left(\boldsymbol{q}_{n+1}\right) \ddot{\boldsymbol{q}}_{n+1}\left(\boldsymbol{q}_{n+1}\right)+\boldsymbol{h}\left(\boldsymbol{q}_{n+1}, \dot{\boldsymbol{q}}_{n+1}\left(\boldsymbol{q}_{n+1}\right)\right)-\boldsymbol{\tau}_{n+1}
$$

From the above equation, the Jacobian matrix obtained by differentiating $\boldsymbol{e}$ with respect to $\boldsymbol{q}_{n+1}$ can be calculated as follows.

$$
\begin{aligned}
\frac{\partial \boldsymbol{e}}{\partial \boldsymbol{q}_{n+1}}= & \frac{\partial \boldsymbol{M}\left(\boldsymbol{q}_{n+1}\right)}{\partial \boldsymbol{q}_{n+1}} \ddot{\boldsymbol{q}}_{n+1}\left(\boldsymbol{q}_{n+1}\right)+\boldsymbol{M}\left(\boldsymbol{q}_{n+1}\right) \frac{\partial \ddot{\boldsymbol{q}}_{n+1}}{\partial \boldsymbol{q}_{n+1}} \\
& +\frac{\partial \boldsymbol{h}\left(\boldsymbol{q}_{n+1}, \dot{\boldsymbol{q}}_{n+1}\left(\boldsymbol{q}_{n+1}\right)\right)}{\partial \boldsymbol{q}_{n+1}}+\frac{\partial \boldsymbol{h}\left(\boldsymbol{q}_{n+1}, \dot{\boldsymbol{q}}_{n+1}\left(\boldsymbol{q}_{n+1}\right)\right)}{\partial \dot{\boldsymbol{q}}_{n+1}} \frac{\partial \dot{\boldsymbol{q}}_{n+1}}{\partial \boldsymbol{q}_{n+1}} \\
= & \boldsymbol{\beta}^{\prime} \boldsymbol{M}\left(\boldsymbol{q}_{n+1}\right)+\boldsymbol{\gamma}^{\prime} \boldsymbol{D}^{t}\left(\boldsymbol{q}_{n+1}\right)+\boldsymbol{K}^{t}\left(\boldsymbol{q}_{n+1}\right) \equiv \boldsymbol{S}\left(\boldsymbol{q}_{n+1}\right)
\end{aligned}
$$

where $\boldsymbol{K}^{t}$ and $\boldsymbol{D}^{t}$ are matrices defined as follows.

$$
\boldsymbol{K}^{t}\left(\boldsymbol{q}_{n+1}\right) \equiv \frac{\partial \boldsymbol{M}\left(\boldsymbol{q}_{n+1}\right)}{\partial \boldsymbol{q}_{n+1}} \ddot{\boldsymbol{q}}_{n+1}\left(\boldsymbol{q}_{n+1}\right)+\frac{\partial \boldsymbol{h}\left(\boldsymbol{q}_{n+1}, \dot{\boldsymbol{q}}_{n+1}\left(\boldsymbol{q}_{n+1}\right)\right)}{\partial \boldsymbol{q}_{n+1}}, \boldsymbol{D}^{t}\left(\boldsymbol{q}_{n+1}\right) \equiv \frac{\partial \boldsymbol{h}\left(\boldsymbol{q}_{n+1}, \dot{\boldsymbol{q}}_{n+1}\left(\boldsymbol{q}_{n+1}\right)\right)}{\partial \dot{\boldsymbol{q}}_{n+1}}
$$

If $\boldsymbol{S}\left(\boldsymbol{q}_{n+1}\right)$ can be calculated, $\boldsymbol{q}_{n+1}, \dot{\boldsymbol{q}}_{n+1}$ and $\ddot{\boldsymbol{q}}_{n+1}$ at time $t=t_{n+1}$ can be obtained by repeating the following calculation until $\boldsymbol{e}\left(\boldsymbol{q}_{n+1}\right)=\mathbf{0}$ is satisfied within the margin of error.

$\boldsymbol{S}\left(\boldsymbol{q}_{n+1}^{(k)}\right) \Delta \boldsymbol{q}_{n+1}^{(k)}=-\boldsymbol{e}^{(k)}, \boldsymbol{q}_{n+1}^{(k+1)}=\boldsymbol{q}_{n+1}^{(k)}+\Delta \boldsymbol{q}_{n+1}^{(k)}, \dot{\boldsymbol{q}}_{n+1}^{(k+1)}=\dot{\boldsymbol{q}}_{n+1}^{(k)}+\gamma^{\prime} \Delta \boldsymbol{q}_{n+1}^{(k)}, \ddot{\boldsymbol{q}}_{n+1}^{(k+1)}=\ddot{\boldsymbol{q}}_{n+1}^{(k)}+\beta^{\prime} \Delta \boldsymbol{q}_{n+1}^{(k)}$ 


\subsection{Acceleration of generalized- $\alpha$ method by recursive algorithm}

In this study, we propose a Jacobian matrix calculation method with high calculation efficiency that takes advantage of the characteristics of the recursive algorithm, and introduce it into the generalized- $\alpha$ method to speed up the calculation. From Eq. (14), it can be seen that $\boldsymbol{K}^{t}$ and $\boldsymbol{D}^{t}$ can be expressed as follows.

$$
\boldsymbol{K}^{t}\left(\boldsymbol{q}_{n+1}\right)=\left.\frac{\partial}{\partial \boldsymbol{q}}\{\boldsymbol{M}(\boldsymbol{q}) \ddot{\boldsymbol{q}}+\boldsymbol{h}(\boldsymbol{q}, \dot{\boldsymbol{q}})\}\right|_{t=t_{n+1}}=\left.\frac{\partial \boldsymbol{\tau}}{\partial \boldsymbol{q}}\right|_{t=t_{n+1}}, \boldsymbol{D}^{t}\left(\boldsymbol{q}_{n+1}\right)=\left.\frac{\partial}{\partial \dot{\boldsymbol{q}}}\{\boldsymbol{M}(\boldsymbol{q}) \ddot{\boldsymbol{q}}+\boldsymbol{h}(\boldsymbol{q}, \dot{\boldsymbol{q}})\}\right|_{t=t_{n+1}}=\left.\frac{\partial \boldsymbol{\tau}}{\partial \dot{\boldsymbol{q}}}\right|_{t=t_{n+1}}
$$

Since the matrix $\boldsymbol{K}^{t}$ is the partial derivative of the inverse dynamics relationship with respect to $\boldsymbol{q}$, it can be obtained by the following calculation.

1) Perform the following calculations from $i=1$ to $N$ and $k=1$ to $N$.

$$
\begin{aligned}
\frac{\partial \boldsymbol{v}_{i}}{\partial q_{k}} & =\boldsymbol{D}_{i} \frac{\partial \boldsymbol{v}_{i-1}}{\partial q_{k}}+\frac{\partial \boldsymbol{D}_{i}}{\partial q_{k}} \boldsymbol{v}_{i-1} \\
\frac{\partial \boldsymbol{a}_{i}}{\partial q_{k}} & =\boldsymbol{D}_{i} \frac{\partial \boldsymbol{a}_{i-1}}{\partial q_{k}}+\frac{\partial \boldsymbol{D}_{i}}{\partial q_{k}} \boldsymbol{a}_{i-1}+\frac{\partial \boldsymbol{\beta}_{i}}{\partial q_{k}}
\end{aligned}
$$

2) Perform the following calculations from $i=N$ to 1 and $k=1$ to $N$.

$$
\begin{aligned}
\frac{\partial \boldsymbol{Q}_{i}^{J}}{\partial q_{k}} & =\boldsymbol{M}_{i} \frac{\partial \boldsymbol{a}_{i}}{\partial q_{k}}+\frac{\partial \boldsymbol{h}_{i}}{\partial q_{k}}-\frac{\partial \boldsymbol{Q}_{i}^{O}}{\partial q_{k}}+\boldsymbol{D}_{i+1}^{T} \frac{\partial \boldsymbol{Q}_{i+1}^{J}}{\partial q_{k}}+\frac{\partial \boldsymbol{D}_{i+1}^{T}}{\partial q_{k}} \boldsymbol{Q}_{i+1}^{J} \\
\frac{\partial \tau_{i}}{\partial q_{k}} & =\boldsymbol{J}_{i}^{T} \frac{\partial \boldsymbol{Q}_{i}^{J}}{\partial q_{k}}+\frac{\partial \bar{Q}_{i}}{\partial q_{k}}
\end{aligned}
$$

These recurrence formulas are obtained by partially differentiating Eqs. (1), (2) and Eqs. (4), (5) with respect to $q_{k}$. Since $\partial \boldsymbol{D}_{i} / \partial q_{k}, \partial \boldsymbol{\beta}_{i} / \partial q_{k}$ and $\partial \boldsymbol{h}_{i} / \partial q_{k}$ appearing in the equation can be calculated in advance, by starting from a known initial value and solving the above recurrence formula in sequence, $\partial \boldsymbol{\tau} / \partial \boldsymbol{q}=\boldsymbol{K}^{t}$ can be obtained without any differential calculation.

Similarly, the matrix $\boldsymbol{D}^{t}=\partial \boldsymbol{\tau} / \partial \dot{\boldsymbol{q}}$ can be calculated as follows.

1) Perform the following calculations from $i=1$ to $N$ and $k=1$ to $N$.

$$
\begin{aligned}
& \frac{\partial \boldsymbol{v}_{i}}{\partial \dot{q_{k}}}=\boldsymbol{D}_{i} \frac{\partial \boldsymbol{v}_{i-1}}{\partial \dot{q}_{k}} \\
& \frac{\partial \boldsymbol{a}_{i}}{\partial \dot{q}_{k}}=\boldsymbol{D}_{i} \frac{\partial \boldsymbol{a}_{i-1}}{\partial \dot{q}_{k}}+\frac{\partial \boldsymbol{\beta}_{i}}{\partial \dot{q}_{k}}
\end{aligned}
$$

2) Perform the following calculations from $i=N$ to 1 and $k=1$ to $N$.

$$
\begin{aligned}
\frac{\partial \boldsymbol{Q}_{i}^{J}}{\partial \dot{q}_{k}} & =\boldsymbol{M}_{i} \frac{\partial \boldsymbol{a}_{i}}{\partial \dot{q}_{k}}+\frac{\partial \boldsymbol{h}_{i}}{\partial \dot{q}_{k}}-\frac{\partial \boldsymbol{Q}_{i}^{O}}{\partial \dot{q}_{k}}+\boldsymbol{D}_{i+1}^{T} \frac{\partial \boldsymbol{Q}_{i+1}^{J}}{\partial \dot{q}_{k}} \\
\frac{\partial \tau_{i}}{\partial \dot{q}_{k}} & =\boldsymbol{J}_{i}^{T} \frac{\partial \boldsymbol{Q}_{i}^{J}}{\partial \dot{q}_{k}}+\frac{\partial \bar{Q}_{i}}{\partial \dot{q}_{k}}
\end{aligned}
$$

These recurrence formulas are obtained by partially differentiating Eqs. (1), (2) and Eqs. (4), (5) with respect to $\dot{q}_{k}$. Since $\partial \boldsymbol{\beta}_{i} / \partial \dot{q}_{k}$ and $\partial \boldsymbol{h}_{i} / \partial \dot{q}_{k}$ appearing in the equation can be calculated in advance, by starting from a known initial value and solving the above recurrence formula in sequence, $\partial \boldsymbol{\tau} / \partial \dot{\boldsymbol{q}}=\boldsymbol{D}^{t}$ can be obtained without any differential calculation.

Since $\boldsymbol{M}\left(\boldsymbol{q}_{n+1}\right)$ can be calculated efficiently by the method explained in section 3, the Jacobian matrix $\boldsymbol{S}\left(\boldsymbol{q}_{n+1}\right)$ in Eq. (14) can be calculated quickly and accurately if $\boldsymbol{K}^{t}\left(\boldsymbol{q}_{n+1}\right)$ and $\boldsymbol{D}^{t}\left(\boldsymbol{q}_{n+1}\right)$ are obtained by the above calculation procedure. However, in order to perform dynamics computations, it is necessary to calculate $\bar{Q}_{i}$ in Eq. (5), and in order to calculate the Jacobian matrix, it is 
necessary to obtain $\partial \bar{Q}_{i} / \partial q_{k}$ in Eq. (21) and $\partial \bar{Q}_{i} / \partial \dot{q}_{k}$ in Eq. (25). In the following, the calculation methods of $\bar{Q}_{i}, \partial \bar{Q}_{i} / \partial q_{k}$ and $\partial \bar{Q}_{i} / \partial \dot{q}_{k}$ in the case of the Voigt model are summarized in section 5 . On the other hand, the Maxwell model is not easy, so in section 6, we first examine the analysis method of the Maxwell model for a simple 1DOF system. Then, using the results, we propose the calculation methods of $\bar{Q}_{i}, \partial \bar{Q}_{i} / \partial q_{k}$ and $\partial \bar{Q}_{i} / \partial \dot{q}_{k}$ in the case of the Maxwell model in section 7.

\section{CALCULATION METHOD FOR VOIGT MODEL}

The Voigt model can express creep, which is one of the viscoelastic properties. In this chapter, we explain the calculation methods of $\bar{Q}_{i}, \partial \bar{Q}_{i} / \partial q_{k}$ and $\partial \bar{Q}_{i} / \partial \dot{q}_{k}$ in the case of the Voigt model, and confirm the effectiveness of the proposed method by numerical simulation.

\subsection{Method for computiong $\bar{Q}_{i}, \partial \bar{Q}_{i} / \partial q_{k}$ and $\partial \bar{Q}_{i} / \partial \dot{q}_{k}$}

In the case of the Voigt model shown in Fig. 5 (a), the reaction torque $\bar{Q}_{i}$ of the torque $Q_{i}$ applied to the joint $i$ can be expressed by the following equation.

$$
\bar{Q}_{i}=k_{i}\left(q_{i}-q_{i}^{0}\right)+c_{i} \dot{q}_{i}
$$

where $q_{i}^{0}$ is the angle at which the spring has a natural length. From the above equation, it can be seen that $\partial \bar{Q}_{i} / \partial q_{k}$ and $\partial \bar{Q}_{i} / \partial \dot{q}_{k}$ can be calculated as follows.

$$
\frac{\partial \bar{Q}_{i}}{\partial q_{k}}=\left\{\begin{array}{cc}
k_{i} & (k=i) \\
0 & (k \neq i)
\end{array}, \quad \frac{\partial \bar{Q}_{i}}{\partial \dot{q}_{k}}=\left\{\begin{array}{cc}
c_{i} & (k=i) \\
0 & (k \neq i)
\end{array}\right.\right.
$$

\subsection{Verification by numerical simulation}

Here, we consider the case where a 2-link manipulator composed of rubber links as shown in Fig. 2 is modeled as shown in Fig. 3 and a Voigt model is added to the joint. The following reference trajectories are given to the 2-link manipulator shown in Fig. 2.

$$
\left\{\begin{array}{l}
\theta_{1}(t)=-\frac{\pi}{4} \cos \left(\frac{\pi}{t_{f}} t\right)+\frac{\pi}{4} \\
\theta_{2}(t)=\frac{\pi}{4} \cos \left(\frac{\pi}{t_{f}} t\right)-\frac{\pi}{4}
\end{array} \quad\left(0 \leq t \leq t_{f}\right)\right.
$$

where $t_{f}$ is the operating time. First, assuming that the two links are rigid bodies, inverse dynamics calculation is performed to obtain the joint driving torques. Then, those driving torques are applied to the 1st and 3rd joints of the model shown in Fig. 3, forward dynamics calculations are conducted, and dynamics simulations that take viscoelasticity into consideration are performed. We set $l_{1}=$ $l_{2}=l_{3}=l_{4}=0.1 \mathrm{~m}, m_{1}=m_{2}=m_{3}=m_{4}=0.01840 \mathrm{~kg}, I_{1}=I_{2}=I_{3}=I_{4}=1.54866 \times 10^{-5} \mathrm{kgm}^{2}$, $k_{1}=k_{3}=0.0001 \mathrm{Nm} / \mathrm{rad}, \quad k_{2}=k_{4}=8000.0 \mathrm{Nm} / \mathrm{rad}, \quad c_{1}=c_{3}=0.0, \quad c_{2}=c_{4}=0.2 \mathrm{Nms} / \mathrm{rad}$ and $t_{f}=2 \mathrm{~s}$ in the simulation.

Let us consider the solution that computed by the Runge-Kutta method with the sufficiently small time step $h=6.66667 \times 10^{-5}$ as the true solution of this problem. Figure 6 shows the errors of the proposed method and the Runge-Kutta method with respect to the reference solution. The Runge-Kutta method has high accuracy, but since it is an explicit method, it is easily destabilized and diverged when $h>1.0 \times 10^{-4}$. On the other hand, the proposed method is based on the generalized- $\alpha$ method which is an implicit method that has excellent stability, it was possible to obtain a solution without divergence even if $h$ is increased.

Figure 7 shows the calculation time required to simulate a physical phenomenon for 2 seconds using the Runge-Kutta method and the proposed method (CPU: Intel (R) Core (TM) i7-7700 CPU @ 3.60GHz, Compiler: Visual $\mathrm{C}++$ ). From the figure, the calculation time is also significantly shortened, and it can be confirmed that the calculation time is faster than the Runge-Kutta method when compared with the same $h$. Furthermore, even if the time step $h$ is large, it does not become 


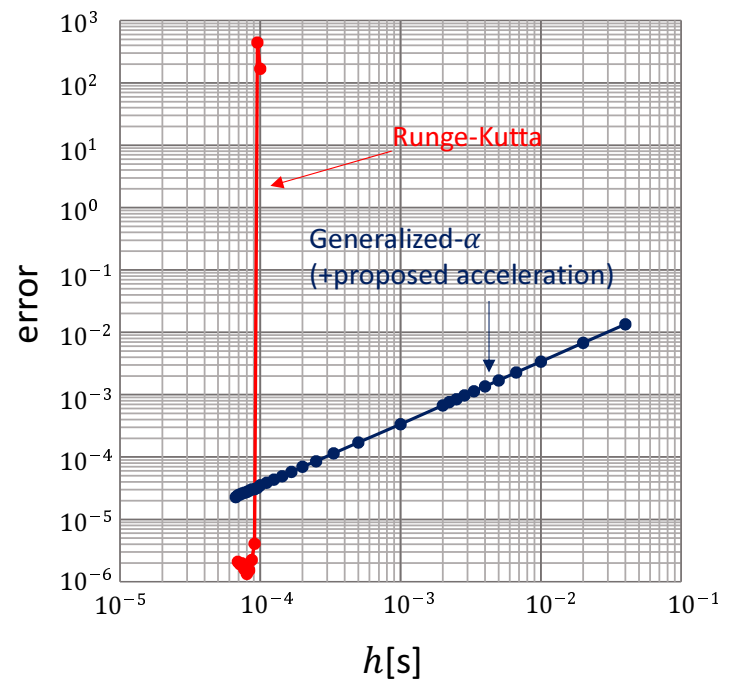

Figure 6. Comparison of integration error (Voigt model)

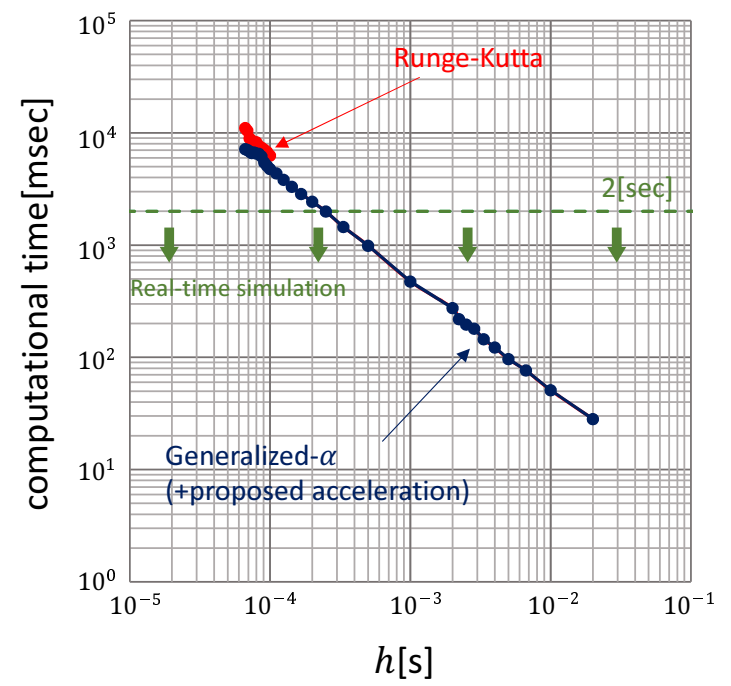

Figure 7. Comparison of computational time (Voigt model)

unstable, so the calculation can be speeded up within the range that satisfies the required accuracy. In this problem, when $h>2.5 \times 10^{-4}$, the calculation time is less than 2 seconds, and real-time calculation can be achieved.

From the above, it was confirmed that when the Voigt model is added to the joint, high-speed and stable dynamics simulation is possible by the proposed method.

\section{EXAMINATION OF ANALYSIS METHOD OF MAXWELL MODEL}

The Voigt model can express creep, which is one of the important properties of viscoelastic bodies, but it is necessary to be able to handle the Maxwell model in order to express stress relaxation, which is another important property. However, in the Maxwell model, it is not easy to calculate $\bar{Q}_{i}$, and the calculation method of its partial derivative has not been established. So far, when calculating the Maxwell model, the equation of motion was often converted into a state equation and the numerical integration method developed for the first-order differential equation was applied. However, in order to introduce the Maxwell model into the calculation methods proposed in sections 3 and 4 , it is necessary to be able to apply the generalized- $\alpha$ method in the form of the equations of motion. Therefore, in this section, the calculation method is first developed for a simple 1DOF system. Then, in section 7, the calculation method is extended to the multibody system.

\subsection{Conventional method based on state equations}

First, the commonly used calculation method will be explained. Let us denote the mass is $M$, the displacement is $q(t)$, and the external force is $Q(t)$ for the 1DOF Maxwell model shown in Fig. 8, then the equation of motion can be written by

$$
M \ddot{q}(t)=Q(t)
$$

If the displacement of the spring and damper alone is denoted by $q_{k}(t)$ and $q_{c}(t)$ respectively, then the following relationship holds.

$$
q(t)=q_{k}(t)+q_{c}(t)
$$




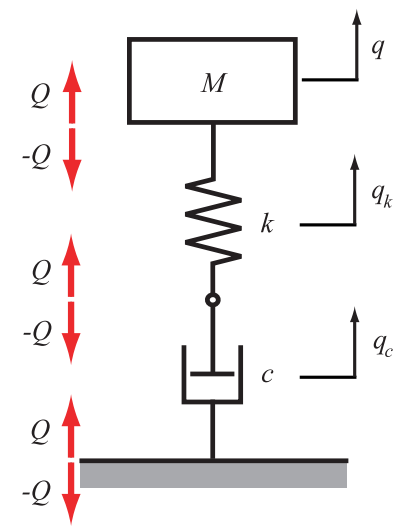

Figure 8. 1-DOF Maxwell model

On the other hand, if the spring constant is $k$ and the damping coefficient is $c$, the external force $Q(t)$ can be expressed in the following two ways.

$$
\begin{aligned}
& Q(t)=-k q_{k}(t) \\
& Q(t)=-c \dot{q}_{c}(t)
\end{aligned}
$$

From Eqs. (29), (31) and (32), the following relationship can be obtained.

$$
\begin{aligned}
& \dot{q}_{k}(t)=-\frac{M}{k} \dddot{q}(t) \\
& \dot{q}_{c}(t)=-\frac{M}{c} \ddot{q}(t)
\end{aligned}
$$

By differentiating Eq. (30) with respect $t$ and substituting Eqs. (33) and (34) leads to

$$
\dot{q}(t)=\dot{q}_{k}(t)+\dot{q}_{c}(t)=-\frac{M}{k} \dddot{q}(t)-\frac{M}{c} \ddot{q}(t)
$$

That is, the following relationship is obtained.

$$
\dddot{q}(t)=-\frac{k}{M} \dot{q}(t)-\frac{k}{c} \ddot{q}
$$

Define the state variables as $x_{1}(t)=q(t), x_{2}(t)=\dot{q}(t), x_{3}(t)=\ddot{q}(t)$, then the following state equations can be obtained.

$$
\begin{aligned}
& \dot{x}_{1}(t)=x_{2}(t) \\
& \dot{x}_{2}(t)=x_{3}(t) \\
& \dot{x}_{3}(t)=-\frac{k}{M} x_{2}(t)-\frac{k}{c} x_{3}(t)
\end{aligned}
$$

By applying the numerical integration method developed for the first-order differential equations such as the Runge-Kutta method to the state equations, the time response $q(t)$ can be calculated.

\subsection{Proposed method based on equations of motion}

In order to introduce the Maxwell model into the calculation methods proposed in sections 3 and 4 , it is necessary to enable numerical integration by the generalized- $\alpha$ method in the form of the equations of motion without converting it to the state equations.

By differentiating Eq. (30) with respect $t$ and substituting Eqs. (31) and (32) leads to

$$
\dot{q}(t)=\dot{q}_{k}(t)+\dot{q}_{c}(t)=-\frac{1}{k} \dot{Q}(t)-\frac{1}{c} Q(t)
$$


That is, the following differential equation is obtained.

$$
\dot{Q}(t)+\frac{k}{c} Q(t)=-k \dot{q}(t)
$$

Define the initial value of $Q(t)$ is $Q_{0}$, then the solution of this differential equation is derived as

$$
Q(t)=-e^{-\frac{k}{c} t}\left\{\int_{0}^{t} e^{\frac{k}{c} \tau} k \dot{q}(\tau) d \tau-Q_{0}\right\}
$$

When the equation of motion (29) and the external force of Eq. (42) are discretized by the time step $h$, it can be expressed as the following equation at $t=t_{n+1}$.

$$
\begin{aligned}
M \ddot{q}_{n+1} & =Q_{n+1} \\
Q_{n+1} & =-e^{-\frac{k}{c}\{(n+1) h\}}\left\{\sum_{j=0}^{n+1} e^{\frac{k}{c}(j h)} k \dot{q}_{j} h-Q_{0}\right\}
\end{aligned}
$$

Furthermore, by arranging the Eq. (44), the following recurrence formula can be obtained.

$$
\begin{aligned}
Q_{n+1} & =-e^{-\frac{k}{c}\{(n+1) h\}}\left\{\sum_{j=0}^{n} e^{\frac{k}{c}(j h)} k \dot{q}_{j} h-Q_{0}+e^{\frac{k}{c}\{(n+1) h\}} k \dot{q}_{n+1} h\right\} \\
& =-k \dot{q}_{n+1} h+e^{-\frac{k}{c} h}\left[-e^{-\frac{k}{c}(n h)}\left\{\sum_{j=0}^{n} e^{\frac{k}{c}(j h)} k \dot{q}_{j} h-Q_{0}\right\}\right] \\
& =-k \dot{q}_{n+1} h+e^{-\frac{k}{c} h} Q_{n}
\end{aligned}
$$

On the other hand, Newmark's integral formula is expressed as follows.

$$
\begin{aligned}
& \dot{q}_{n+1}=\dot{q}_{n}+h(1-\gamma) \ddot{q}_{n}+h \gamma \ddot{q}_{n+1} \\
& q_{n+1}=q_{n}+h \dot{q}_{n}+h^{2}\left(\frac{1}{2}-\beta\right) \ddot{q}_{n}+h^{2} \beta \ddot{q}_{n+1}
\end{aligned}
$$

Here, if we define $\Delta q=q_{n+1}-q_{n}$, the following relationship can be obtained.

$$
\begin{aligned}
q_{n+1} & =q_{n}+\Delta q \\
\dot{q}_{n+1} & =\left(1-\frac{\gamma}{2 \beta}\right) h \ddot{q}_{n}+\frac{\gamma}{\beta h} \Delta q \\
\ddot{q}_{n+1} & =\left(1-\frac{1}{2 \beta}\right) \ddot{q}_{n}-\frac{1}{\beta h} \dot{q}_{n}+\frac{1}{\beta h^{2}} \Delta q
\end{aligned}
$$

In the generalized- $\alpha$ method, the equation of motion is modified as follows in order to improve the numerical damping characteristics.

$$
\begin{aligned}
M \ddot{q}^{*} & =Q^{*} \\
\ddot{q}^{*} & =\left(1-\alpha_{m}\right) \ddot{q}_{n+1}+\alpha_{m} \ddot{q}_{n} \\
Q^{*} & =\left(1-\alpha_{f}\right) Q_{n+1}+\alpha Q_{n}
\end{aligned}
$$

Substituting Eqs. (52), (53) and Eqs. (48) to (50) into Eq. (51), we can obtain the following equation.

$$
\begin{aligned}
\left\{\frac{1-\alpha_{m}}{h^{2} \beta\left(1-\alpha_{f}\right)} M+\frac{\gamma}{\beta} k\right\} \Delta q= & {\left[\left(e^{-\frac{k}{c} h}+\frac{\alpha_{f}}{1-\alpha_{f}}\right) Q_{n}-\frac{1-\alpha_{m}}{1-\alpha_{f}} M\left\{\left(1-\frac{1}{2 \beta}\right) \ddot{q}_{n}-\frac{1}{\beta h} \dot{q}_{n}\right\}\right.} \\
& \left.-\frac{\alpha_{m}}{1-\alpha_{f}} M \ddot{q}_{n}-k\left\{\left(1-\frac{\gamma}{2 \beta}\right) h^{2} \ddot{q}_{n}+\left(1-\frac{\gamma}{\beta}\right) h \dot{q}_{n}\right\}\right]
\end{aligned}
$$

Since the coefficient of $\Delta q$ and right hand side in the above equation are known, $\Delta q$ can be calculated. Then, by substituting $\Delta q$ into Eqs (48) to (50), $q_{n+1}, \dot{q}_{n+1}$ and $\ddot{q}_{n+1}$ can be calculated. Furthermore, by substituting $\dot{q}_{n+1}$ into Eq. (45), $Q_{n+1}$ can be obtained. By repeating the above procedure, the time response $q(t)$ can be calculated. 


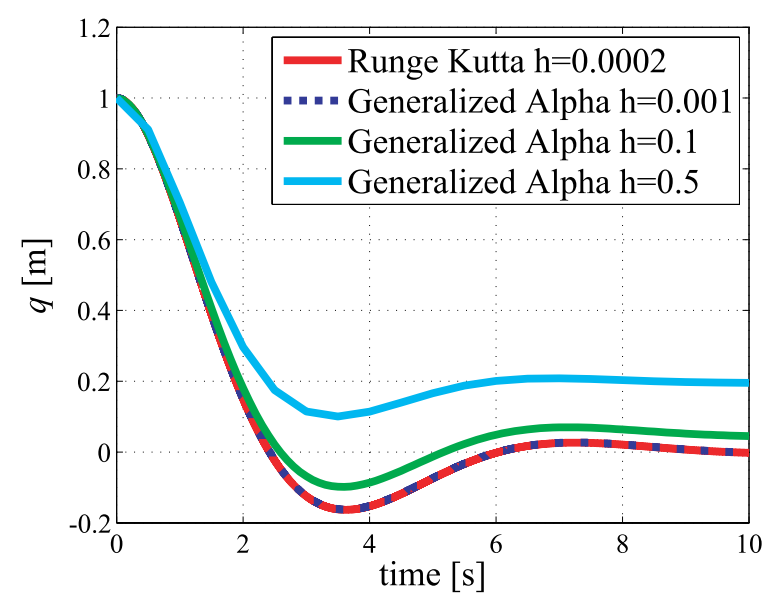

Figure 9. Comparison of time response

\subsection{Verification by numerical simulation}

Here, we compare the proposed method explained in Section 6.2 with the conventional method explained in Section 6.1. As an example, the dynamics simulation was performed with the parameters set to $M=1 \mathrm{~kg}, D=1 \mathrm{Ns} / \mathrm{m}, K=1 \mathrm{~N} / \mathrm{m}, Q_{0}=-1.0 \mathrm{~N}, q(0)=1.0 \mathrm{~m}, \dot{q}(0)=0 \mathrm{~m} / \mathrm{s}$ and $\ddot{q}(0)=Q_{0} / M=-1.0 \mathrm{~m} / \mathrm{s}^{2}$. The red line in Fig. 9 shows the result of solving by applying the conventional method and the Runge-Kutta method. Since the time step $h$ is set sufficiently small as $h=0.0002$, it is considered that a solution close to the true one is obtained. Therefore, the validity of the proposed method is verified using this solution as a reference solution. The result of the calculation with $h=0.001$ by the proposed method is shown by the blue dashed line in Fig. 9 . From the figure, it can be confirmed that the blue dashed line overlaps with the red line and the calculation can be performed accurately. Since the proposed method uses the generalized- $\alpha$ method, which is an implicit numerical integration method, it did not diverge even if it was increased to $h=0.1,0.5$. From the above, the validity of the proposed calculation method was confirmed.

\section{CALCULATION METHOD FOR MAXWELL MODEL}

In this section, the analysis method of the Maxwell model developed for 1DOF system in section 6 is extended to multibody systems. We formulate the calculation methods of $\bar{Q}_{i}, \partial \bar{Q}_{i} / \partial q_{k}$ and $\partial \bar{Q}_{i} / \partial \dot{q}_{k}$ required when using the methods proposed in sections 3 and 4 for the Maxwell model.

\subsection{Method for computiong $\bar{Q}_{i}, \partial \bar{Q}_{i} / \partial q_{k}$ and $\partial \bar{Q}_{i} / \partial \dot{q}_{k}$}

In the case of the Maxwell model shown in Fig. 5 (b), the torque $Q_{i}(t)$ applied to the joint $i$ can be calculated from Equation (42) as follows.

$$
Q_{i}(t)=-e^{-\frac{k_{i}}{c_{i}} t}\left\{\int_{0}^{t} e^{\frac{k_{i}}{c_{i}} \tau} k_{i} \dot{q}_{i}(\tau) d \tau-Q_{i}^{0}\right\}
$$

where $Q_{i}^{0}$ is the initial value of $Q_{i}(t)$. Then, the Eq. (5) can be expressed as follows.

$$
\tau_{i}=\boldsymbol{J}_{i}^{T} \boldsymbol{Q}_{i}^{J}+\bar{Q}_{i}(t), \bar{Q}_{i}(t)=-Q_{i}(t)=e^{-\frac{k_{i}}{c_{i}}}\left\{\int_{0}^{t} e^{\frac{k_{i}}{c_{i}} \tau} k_{i} \dot{q}_{i}(\tau) d \tau-Q_{i}^{0}\right\}
$$

From Eq. (45), it is understood that $Q_{i}(t)$ can be expressed by the following recurrence formula, if it is discretized by the time step $h$ at $t=t_{n}$.

$$
Q_{i}\left(t_{n}\right)=-k_{i} \dot{q}_{i}\left(t_{n}\right) h+e^{-\frac{k_{i}}{c_{i}} h} Q_{i}\left(t_{n-1}\right)
$$


Therefore, $\bar{Q}_{i}(t)$ in Eq. (56) can be calculated by the following recurrence formula.

$$
\bar{Q}_{i}\left(t_{n}\right)=k_{i} \dot{q}_{i}\left(t_{n}\right) h+e^{-\frac{k_{i}}{c_{i}} h} \bar{Q}_{i}\left(t_{n-1}\right)
$$

Next, let us consider the calculation methods of $\partial \bar{Q}_{i} / \partial q_{k}$ and $\partial \bar{Q}_{i} / \partial \dot{q}_{k}$ that required when calculating the Jacobian matrix. By shifting the subscript of Eq. (58) by one and rewriting it for $t=t_{n+1}$, the following equation is obtained.

$$
\bar{Q}_{i}\left(t_{n+1}\right)=k_{i} \dot{q}_{i}\left(t_{n+1}\right) h+e^{-\frac{k_{i}}{c_{i}} h} \bar{Q}_{i}\left(t_{n}\right)
$$

By partially differentiating the above equation, the following relationship can be obtained.

$$
\left.\frac{\partial \bar{Q}_{i}}{\partial q_{i}}\right|_{t=t_{n+1}}=\frac{\partial \bar{Q}_{i}\left(t_{n+1}\right)}{\partial q_{i}\left(t_{n+1}\right)}=0,\left.\frac{\partial \bar{Q}_{i}}{\partial \dot{q}_{i}}\right|_{t=t_{n+1}}=\frac{\partial \bar{Q}_{i}\left(t_{n+1}\right)}{\partial \dot{q}_{i}\left(t_{n+1}\right)}=k_{i} h
$$

That is, $\partial \bar{Q}_{i} / \partial q_{k}$ and $\partial \bar{Q}_{i} / \partial \dot{q}_{k}$ can be calculated as follows.

$$
\frac{\partial \bar{Q}_{i}}{\partial q_{k}}=0, \quad \frac{\partial \bar{Q}_{i}}{\partial \dot{q}_{k}}= \begin{cases}k_{i} h & (k=i) \\ 0 & (k \neq i)\end{cases}
$$

From the above, the calculation method proposed in sections 3 and 4 can be extended to the multibody system in which the Maxwell model is added to the joint.

\subsection{Verification by numerical simulation}

Here, we consider the case where a 2-link manipulator composed of rubber links as shown in Fig. 2 is modeled as shown in Fig. 3 and a Maxwell model is added to the joints. As in section 5.2, the desired trajectory represented by Eq. (28) is given to the 2-link manipulator in Fig. 2, and the inverse dynamics calculation is performed by assuming that the two links are rigid bodies. Then, the obtained joint driving torques are applied to the 1st and 3rd joints of the model shown in Fig. 3 to perform a dynamic simulation considering viscoelasticity. The parameters are set to $l_{1}=l_{2}=$ $l_{3}=l_{4}=0.1 \mathrm{~m}, \quad m_{1}=m_{2}=m_{3}=m_{4}=0.01840 \mathrm{~kg}, \quad I_{1}=I_{2}=I_{3}=I_{4}=1.54866 \times 10^{-5} \mathrm{kgm}^{2}$, $k_{1}=k_{3}=0.0001 \mathrm{Nm} / \mathrm{rad}, k_{2}=5000.0 \mathrm{Nm} / \mathrm{rad}, k_{4}=7000.0 \mathrm{Nm} / \mathrm{rad}, c_{1}=c_{3}=0.0001 \mathrm{Nms} / \mathrm{rad}$, $c_{2}=0.2 \mathrm{Nms} / \mathrm{rad}, \quad c_{4}=0.3 \mathrm{Nms} / \mathrm{rad}$ and $t_{f}=2 \mathrm{~s}$.

Let us consider the solution that computed by the Runge-Kutta method with the sufficiently small time step $h=6.66667 \times 10^{-5}$ as the true solution of this problem. Figure 10 shows the errors of the proposed method and the Runge-Kutta method with respect to the reference solution. The Runge-Kutta method has high accuracy, but since it is an explicit method, it is easily destabilized and diverged when $h>9.52 \times 10^{-5}$. On the other hand, the proposed method is based on the generalized- $\alpha$ method which is an implicit method that has excellent stability, it was possible to obtain a solution without divergence even if $h$ is increased.

Figure 11 shows the calculation time required to simulate a physical phenomenon for 2 seconds using the Runge-Kutta method and the proposed method (CPU: Intel (R) Core (TM) i7-7700 CPU@3.60GHz, Compiler: Visual C ++). From the figure, the calculation time is also significantly shortened, and it can be confirmed that the calculation time is faster than the Runge-Kutta method when compared with the same $h$. Furthermore, even if the time step $h$ is large, it does not become unstable, so the calculation can be speeded up within the range that satisfies the required accuracy. In this problem, when $h>2.5 \times 10^{-4}$, the calculation time is less than 2 seconds, and real-time calculation can be achieved.

From the above, it was confirmed that high-speed and stable dynamical simulation is possible by the proposed method even when the Maxwell model is added to the joint. 


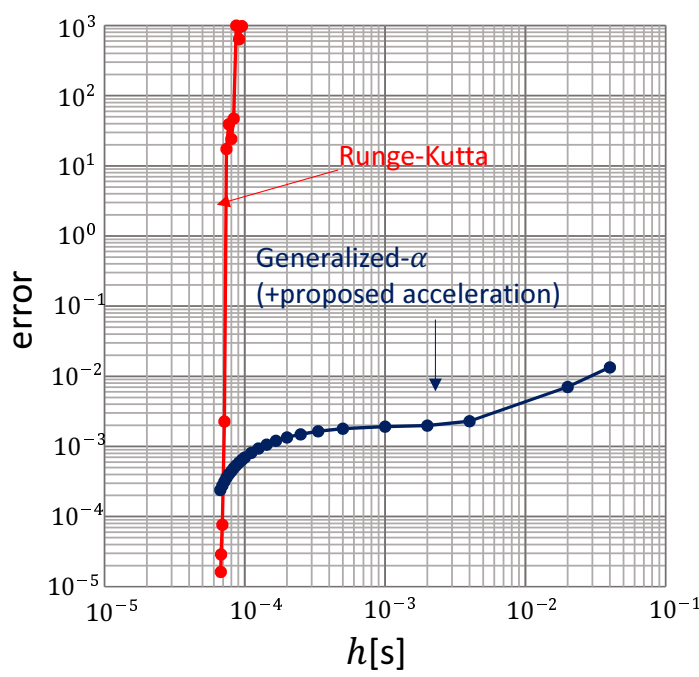
Figure 10. Comparison of integration error
(Maxwell model)

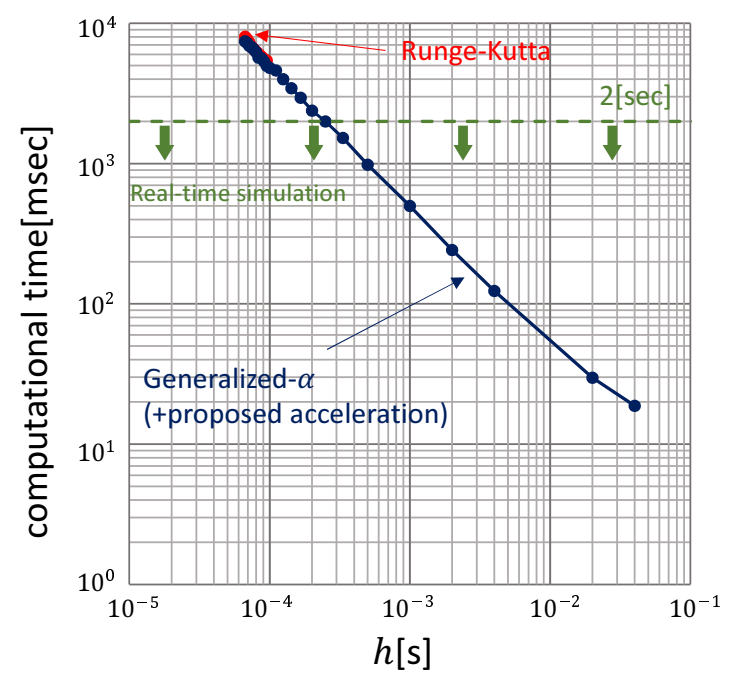

Figure 11. Comparison of computational time (Maxwell model)

\section{CONCLUSIONS}

In this study, we investigated a high-speed and stable dynamic calculation method for soft robot systems containing viscoelastic bodies such as rubber and resin. The viscoelastic bodies were approximated by finite rigid body segments, and they were connected by joints and linear viscoelastic elements such as the Voigt model and Maxwell model to express the viscoelastic properties. Then, the recursive dynamics algorithm was used to speed up the calculation, and the generalized- $\alpha$ method was used to stabilize the numerical integration. In this paper, we examined how to incorporate the Maxwell model into recursive dynamics algorithm and the generalized- $\alpha$ method, and proposed a new method. We applied the proposed method to a 2-link manipulator composed of rubber links and confirmed the effectiveness of the proposed method. In our future work, we will extend the proposed method to the generalized Maxwell model as shown in Fig. 1 (c) in order to reproduce the actual behavior of the viscoelastic body more accurately.

\section{REFERENCES}

[1] Laschi, C., eds.: Soft Robotics, Trends, Applications and Challenges, Proceedings of the Soft Robotics Week, Springer (2016).

[2] Zhang, Y., Tian, Q., Chen, L., Yang, J.: Simulation of a viscoelastic flexible multibody system using absolute nodal coordinate and fractional derivative method, Multibody System Dynamics, 21 (2009) 281-303.

[3] Mohamed, A.A., and Shabana, A.A.: A nonlinear visco-elastic constitutive model for large rotation finite element formulations, Multibody System Dynamics, 26 (2011) 57-79.

[4] Iwamura, M, Sugiyama, H., Sato, K.: Recursive inverse and forward dynamics algorithms for flexible manipulators. Proceedings of the 6th Asian Conference on Multibody Dynamics, USB 9 pages, 2012.

[5] Arnold, M., and Bruls, O.: Convergence of the generalized- $\alpha$ scheme for constrained mechanical systems, Multibody System Dynamics, 18 (2007) 185-202. 